\title{
Surgical Outcomes of Dysphagia Provoked by Diffuse Idiopathic Skeletal Hyperostosis in the Cervical Spine
}

\author{
Young Soo Chung ${ }^{1}$, Ho Yeol Zhang ${ }^{2}$, Yoon $\mathrm{Ha}^{1}$, and Jeong-Yoon Park ${ }^{3}$ \\ ${ }^{1}$ Department of Neurosurgery, Severance Hospital, Yonsei University College of Medicine, Seoul; \\ ${ }^{2}$ Department of Neurosurgery, National Health Insurance Service Ilsan Hospital, Goyang; \\ ${ }^{3}$ Department of Neurosurgery, Gangnam Severance Hospital, Yonsei University College of Medicine, Seoul, Korea
}

Purpose: This study aimed to predict the surgical outcomes of diffuse idiopathic skeletal hyperostosis (DISH)-related dysphagia (DISH-phagia) and to evaluate the importance of prevertebral soft tissue thickness (PVST).

Materials and Methods: In total, 21 surgeries (anterior osteophytectomy or anterior cervical decompression and fixation) were included in this study for DISH-phagia from 2003 to 2019. Clinical outcomes were assessed using the Dysphagia Outcome and Severity Scale (DOSS) preoperatively, at 1 month postoperatively, and last follow up (mean 29.5 months). PVST was measured using lateral plain radiographs. Paired t-test and Spearman's correlation test was used to identify relationships between various PVST indices and DOSS.

Results: Comparisons were made from 17 patients out of 21, in which the record had all of three measurements. The narrowest PVST preoperatively was $2.55 \pm 0.90 \mathrm{~mm}$, with a DOSS score of $4.47 \pm 1.61$, and that at 1 month after surgery was $5.02 \pm 2.33 \mathrm{~mm}$, with a DOSS score of $6.12 \pm 1.32$. At last follow up, PVST and DOSS values were $3.78 \pm 0.92 \mathrm{~mm}$ and $5.82 \pm 1.34$, and three patients experienced symptom relapse. Significant relationships were found between PVST and DOSS at all time points: before surgery $(\mathrm{R}=0.702, p<0.001), 1$ month after surgery $(\mathrm{R}=0.539, p=0.012)$, and last follow up $(\mathrm{R}=0.566, p=0.020)$.

Conclusion: Surgical removal of anterior osteophytes is an effective treatment option for DISH-phagia, and PVST is a useful parameter in DISH-phagia. The goal of DISH surgery should be to remove DISH as much as possible to ensure sufficient PVST postoperatively.

Key Words: Diffuse idiopathic skeletal hyperostosis, dysphagia, osteophyte

\section{INTRODUCTION}

Diffuse idiopathic skeletal hyperostosis (DISH) of the cervical

Received: January 2, 2020 Revised: February 17, 2020

Accepted: February 25, 2020

Co-corresponding authors: Ho Yeol Zhang, MD, PhD, Department of Neurosurgery, National Health Insurance Service IIsan Hospital, 100 IIsan-ro, Ilsandong-gu, Goyang 10444, Korea.

Tel: 82-31-900-6997, Fax: 82-31-900-0049, E-mail: hyzhang@nhimc.or.kr and

Jeong-Yoon Park, MD, PhD, Department of Neurosurgery, Gangnam Severance Hospital, Yonsei University College of Medicine, 211 Eonju-ro, Gangnam-gu, Seoul 06273, Korea.

Tel: 82-2-2019-3390, Fax: 82-2-3461-9229, E-mail: spinepjy@gmail.com

-The authors have no potential conflicts of interest to disclose.

(C) Copyright: Yonsei University College of Medicine 2020

This is an Open Access article distributed under the terms of the Creative Commons Attribution Non-Commercial License (https://creativecommons.org/licenses/ by-nc/4.0) which permits unrestricted non-commercial use, distribution, and reproduction in any medium, provided the original work is properly cited. spine is a common spinal degenerative disease observed in $10 \%$ to $30 \%$ of the general population, ${ }^{1}$ and it rarely manifests as dysphagia. Some have regarded it to contribute to stiffness and restricted segmental motion, ${ }^{2-4}$ although in some cases, mechanical compression of the tracheoesophageal complex can result in dysphagia, hoarseness, and even dyspnea. It is presumed that dysphagia or airway obstruction due to cervical DISH is present in only $0.1 \%$ to $4 \%$ of cases: $:^{5-7}$ Some authors refer to it as DISH-phagia, ${ }^{8}$ which is a neologism of DISH and dysphagia. The prevalence of DISH-phagia is unknown, as many cases are not reported because of unfamiliarity among physicians with its signs.

The disease course and treatment strategy of DISH-phagia are not established. While a few studies have reported longterm surgical outcomes, ${ }^{9-12}$ no statistical analyses have been performed in this context. This study aimed to outline progno- 
sis after surgery and to predict the surgical outcomes of DISHphagia through radiographic measurements and a review of the course of the disease, including recurrence.

\section{MATERIALS AND METHODS}

\section{Patient selection and characteristics}

This retrospective, cross-sectional study included patients who were diagnosed with DISH-phagia. Twenty-four surgeries were performed because of dysphagia with severe cervical DISH from 2003 to 2019 in our three institutions by multiple surgeons. Because of the retrospective study design, the need for informed consent was waived. We obtained study approval from the Institutional Review Board of National Health Insurance Service Ilsan Hospital (IRB number: 2019-02-001).

Inclusion criteria were as follows: 1) diagnosis of cervical DISH by a spine surgeon and radiologists using magnetic resonance imaging and computed tomography, 2) definite esophageal compression on laryngoscope or video fluoroscopic swallowing study (VFSS) (Fig. 1), 3) preoperative Dysphagia Outcome and Severity Scale (DOSS) score $\leq 6,{ }^{13}$ and 4 ) availability of postoperative radiographs after 1 month and last follow up. Exclusion criteria were 1) patients whose anterior osteophyte or hyperostosis protruded at only one level or two levels, which does not fit DISH criteria, ${ }^{14}$ 2) patients who had other medical diseases that are attributable to dysphagia (e.g., stroke, neuromuscular disease, and advanced lung cancer), and 3) patients who had acute fracture of the osteophyte or hyperostosis, because pain can be a confounding factor.

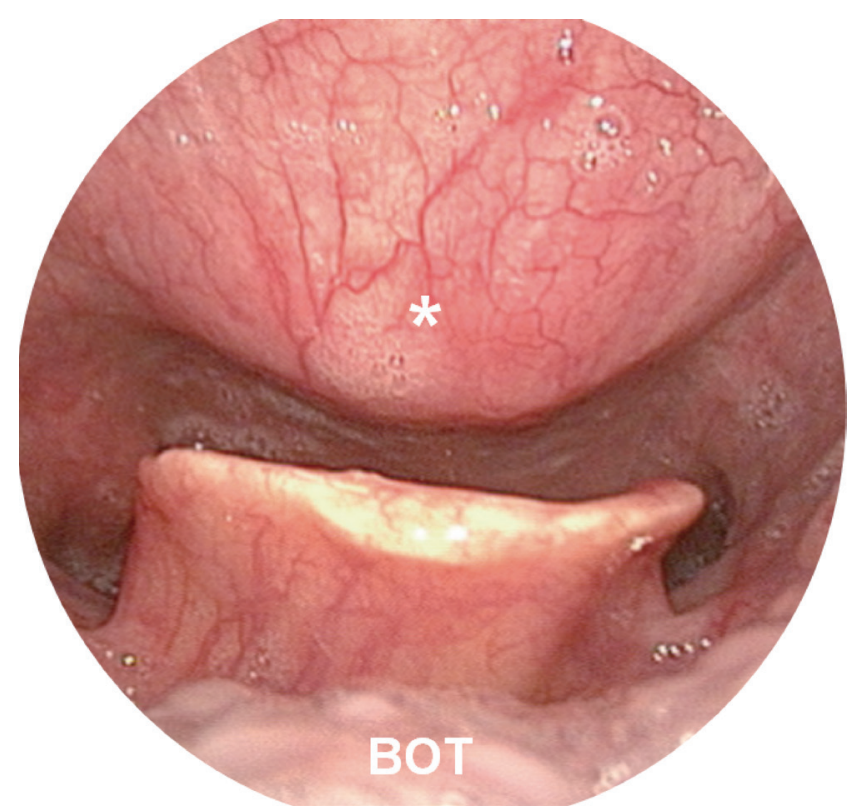

Fig. 1. Laryngoscopic view of severe diffuse idiopathic skeletal hyperostosis. The asterisk indicates the vertebral osteophyte. The hypopharynx and epiglottis are also seen. BOT, base of the tongue.

\section{Measurements and definitions}

The severity of dysphagia was scored according to the DOSS. ${ }^{13}$ In this scale, the patient is scored from 1 to 7 as follows: severe dysphagia that cannot tolerate anything safely (1), moderate severe dysphagia that tolerates at least one consistency safely with maximum assistance (2), moderate dysphagia in which assistance or supervision is absolutely needed (3), mild-moderate dysphagia that requires intermittent supervision with one or two consistencies restricted (4), mild dysphagia that often needs distant assistance (5), inconvenience in swallowing within functional limits (6), and normal in all situations (7). If the last postoperative DOSS score was lower than the 1-month postoperative DOSS, DISH-phagia was regarded as having recurred. Because DOSS scoring ranges from 1 to 7 , we regarded it as a numerical scale, not a categorical scale. Postoperative complications, including hoarseness, dyspnea, motor weakness, were recorded at each outpatient clinic follow up.

Radiographic comparisons were made using true lateral cervical spine plain radiographs. Because the posterior vertebral line (PVL) is well preserved and easily recognized, various parameters in this study were assessed in relation to the PVL, from the C2 lower endplate to the C7 upper endplate. From the upper and lower posterior corners of each vertebra, an imaginary perpendicular line meeting the most anterior portion of the osteophyte was defined as PVL-O (ossified lesion) (Fig. 2A), and the posterior border of the trachea was defined as PVL-T (trachea) (Fig. 2B). Then, prevertebral soft tissue thickness (PVST) was calculated as PVL-T minus PVL-O (Fig. 2C). Since the narrowest preoperative PVST level is thought to be a symptom-provoking lesion, we scrutinized how it changed postoperatively (Fig. 3). Preoperative PVST at each cervical level was compared to data of a normal population from previous reports. Two previous studies (Omercikoglu, et al., ${ }^{15} \mathrm{n}=488$ and Rojas, et al., ${ }^{16} \mathrm{n}=192$ ) were referenced for this comparison. Because only a single measurement was taken at each vertebra in those studies, we selected the lower value of the two measurements (upper or lower endplate) for each cervical spine. All radiographic parameters were measured using the Centricity PACS system (GE Healthcare, Chicago, IL, USA). Two neurosurgeons (Y.S.C. and H.Y.Z.) measured the radiologic parameters independently using the same protocol, and the mean values thereof were obtained. Interobserver reliability was evaluated through a two-way random model.

All radiologic parameters and measurements of DOSS were gathered at 1 month after surgery for all patients. Four patients were lost to follow up, and the rest of the patients ( $\mathrm{n}=17$ ) underwent long-term follow up (median: 29.5 months). For them, the last PVST and DOSS date were investigated.

\section{Surgical technique}

The surgical techniques included anterior osteophytectomy alone using a high-speed drill $(\mathrm{n}=17)$ and anterior osteophy- 

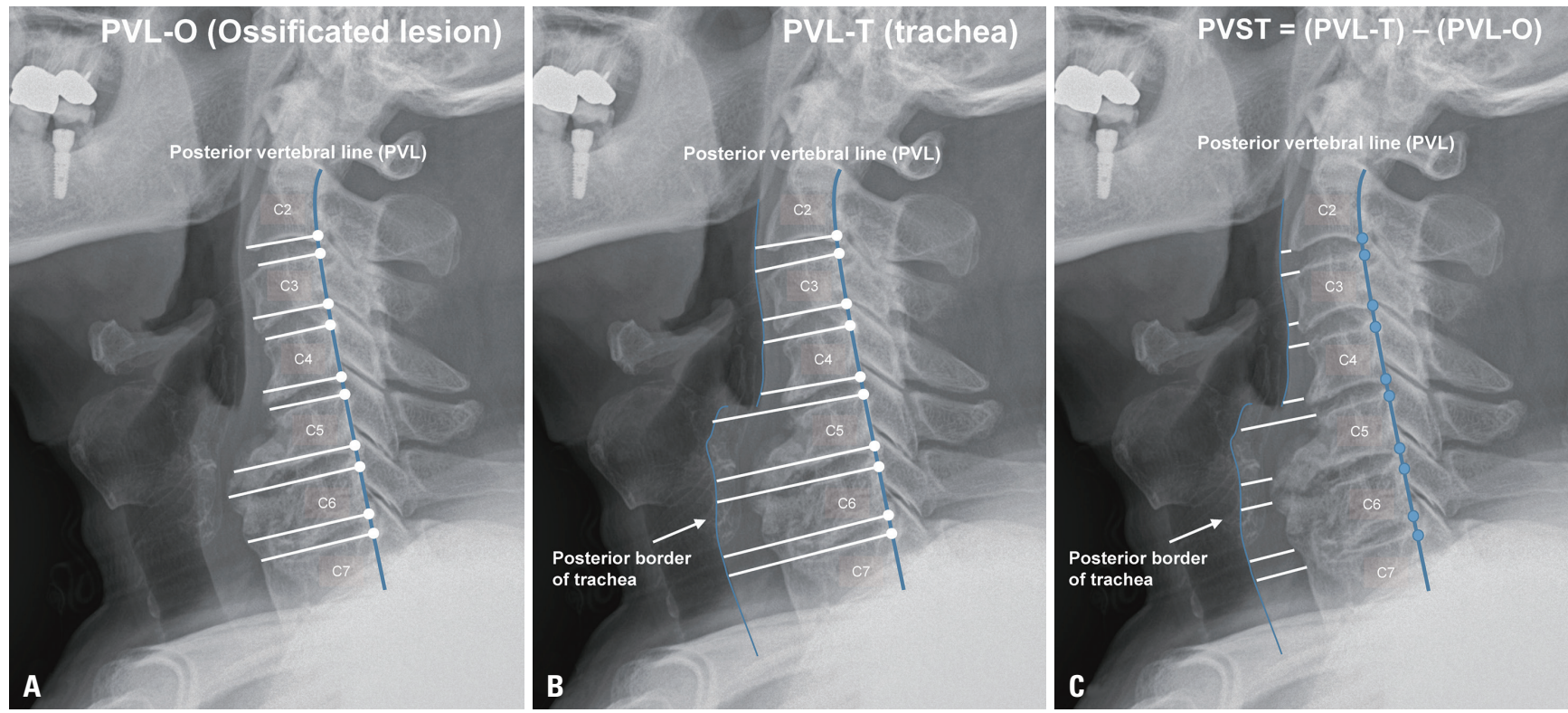

Fig. 2. Radiologic parameters related with diffuse idiopathic skeletal hyperostosis. As PVL alignment is well preserved, distances from the PVL were measured. (A) PVL-O (ossified) is defined as the distance between the PVL and the most anterior portion of the ossified lesion, and each imaginary line is perpendicular to the PVL. (B) PVL-T (trachea) is defined as the distance from the PVL to the posterior border of the trachea, and each imaginary line is perpendicular to the PVL. Both the PVL-0 and PVL-T are measured from the C2 lower endplate to C7 upper endplate. (C) By simple geometric logic, prevertebral soft tissue thickness was calculated as (PVL-T minus PVL-0). PVL, posterior vertebral line.
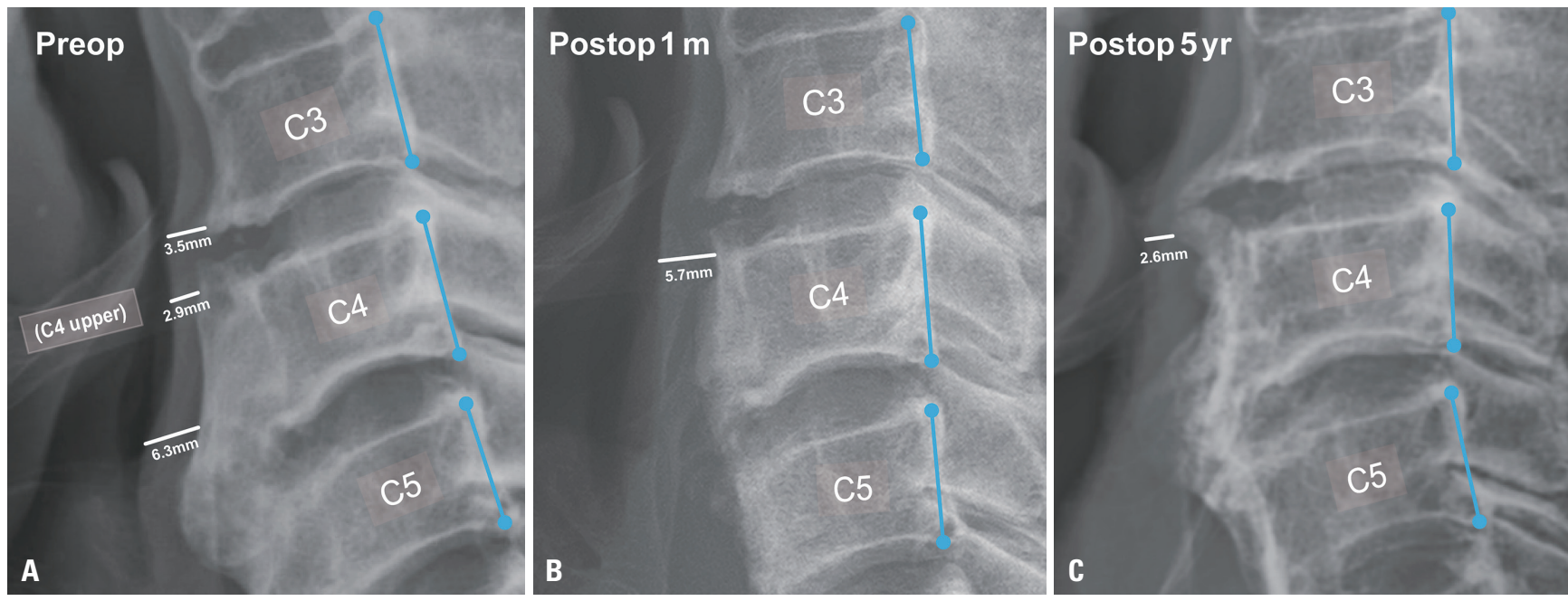

Fig. 3. Change in PVST before and after the surgery. (A) Before surgery, the narrowest PVST $(2.9 \mathrm{~mm})$ was located in the C4 upper endplate, and the preoperative DOSS score was 5. (B) Anterior osteophytectomy was performed, and PVST changed to $5.7 \mathrm{~mm}$ at postoperative 1 month. Symptoms had also improved (DOSS=6). (C) Five years after surgery, bony spur regrowth with a narrowing of PVST (2.6 mm) was noted, and dysphagia occurred again (DOSS=5). PVST, prevertebral soft tissue thickness; DOSS, Dysphagia Outcome and Severity Scale.

tectomy with screw and plate fixation $(\mathrm{n}=4)$. The choice of surgical technique was based on the surgeon's preference. All surgeons tried to remove osteophytes as much as possible within their surgical visualization. We also considered patient comorbidities, such as cervical radiculopathy and instability for plate fixation. Conventional transverse anterolateral skin incisions were used in all patients. In one patient, two parallel transverse incisions were made. The use of an intraoperative $\mathrm{C}$-arm was not standardized. Postoperatively, the amount and range of osteophyte removal were inconsistent. Since the lateral-to-lateral operation range in the surgical field could not be evaluated on plain radiographs, lateral extension depended on palpation and exposure of the cancellous bone.

\section{Statistical analysis}

We used SPSS statistics software, version 25.0 (IBM Corp., Armonk, NY, USA) and GraphPad Prism 8 (GraphPad Inc., San Diego, CA, USA) for analyses. The non-parametric Spearman's 
correlation test was utilized to compare DOSS scores and PVST values. A scatter plot was drawn to visualize the relationship between them. Paired t-test was performed to compare PVST and DOSS in a matched cohort. In all analyses, a $p$ value $<0.05$ was considered indicative of statistical significance.

\section{RESULTS}

Initially, 24 patients were included our study; however, one patient was excluded because an anterior osteophyte was noted only on C6/7, which did not meet the definition of DISH. One patient who had an acute fracture of the C2/3 osteophyte and another patient later determined to have amyotrophic lateral sclerosis were also excluded. Finally, we included 21 cases in this study, which is the largest cohort of DISH-phagia reported to date, except in meta-analyses. Patient characteristics and demographics are described in Table 1.

All patients were men, and 17/21 (81\%) of them were older than 60 years of age (mean age 65.5 years). Four patients (19\%) had accompanied either ankylosing spondylitis (AS) or peripheral enthesopathy. No one showed major surgical complications, such as esophageal injury, airway obstruction, severe disability or death. Postoperative hoarseness was recorded in 3 (14\%) patients, and one (5\%) suffered from sustained hoarseness. One patient (5\%) showed transient right side weakness and recovered after 1 week. Of all 21 surgical cases, 16 (76\%) patients experienced improvement of symptoms after 1 month, whereas 5 (24\%) patients remained the same. For the 17 cases in which long-term surveillance (mean 29.5 months) was available, 3 (18\%) patients reported recurrence of symptoms and a reduction in DOSS, compared with 1 month after surgery, although an additional operation was not performed.

Table 1. Clinical Characteristics of the Patients ( $n=21)$

\begin{tabular}{lc}
\hline \multicolumn{1}{c}{ Characteristic } & Value \\
\hline Age (yr) & 65.5 (range: 55-78) \\
Male sex & $21(100)$ \\
Follow-up period (month) & $29.5 \pm 20.7$ \\
Comorbidities & \\
Hypertension & $8(38.1)$ \\
Diabetes mellitus & $4(19.0)$ \\
OPLL/OLF & $13(61.9)$ \\
Ankylosing spondylitis & $2(9.5)$ \\
$\quad$ Peripheral enthesopathy & $2(9.5)$ \\
Surgical method & \\
Anterior osteophytectomy alone & $17(81.0)$ \\
Anterior osteophytectomy with plate fixation & $4(19.1)$ \\
\hline
\end{tabular}

OPLL, ossification of posterior longitudinal ligament; OLF, ossification of ligamentum flavum; PVST, prevertebral soft tissue thickness; DOSS, Dysphagia Outcome and Severity Scale.

Data are presented as means \pm standard deviations or $\mathrm{n}(\%)$ unless otherwise noticed.
Preoperative average PVST values at each level were $3.2 \mathrm{~mm}$ (C2), $2.9 \mathrm{~mm}$ (C3), $3.6 \mathrm{~mm}$ (C4), $8.8 \mathrm{~mm}$ (C5), $10.6 \mathrm{~mm}$ (C6), and $9.5 \mathrm{~mm}$ (C7) (Fig. 4). The narrowest PVST location before surgery was above the C5 upper level in all patients; of these, 12 (57\%) were at level C3 (C3 upper or lower). For the 17 longterm surveillance cases, mean PVST (preop: $2.55 \mathrm{~mm}$, postop 1 month: $5.02 \mathrm{~mm}$, and last follow up: $3.78 \mathrm{~mm}$ ) and DOSS (4.47, 6.12, and 5.82) are listed in Fig. 5. Improvements in both PVST and DOSS at 1 month after surgery were noted (PVST: from 2.55 to $5.02, p<0.001$, DOSS: from 4.47 to $6.12, p=0.001$ ). Significant regression of PVST was observed in the last follow up, compared to 1 month after surgery (from 5.02 to 3.78, $p=0.016$ ), although deterioration of DOSS was not statistically significant (from 6.12 to $5.82, p=0.096$ ). The total interobserver reliability of the radiologic measurements for the two neurosurgeons was 0.970 , and the intraobserver reliability for measurements of each cervical level ranged from 0.744 to 0.981 . Scatter plots for PVST and DOSS score are provided in Fig. 6. A statistically significant correlation was noted at all times, with $\mathrm{R}$ values ranging from 0.539 to 0.702 . A regression line was marked on each graph, representing positive correlation between PVST and DOSS.

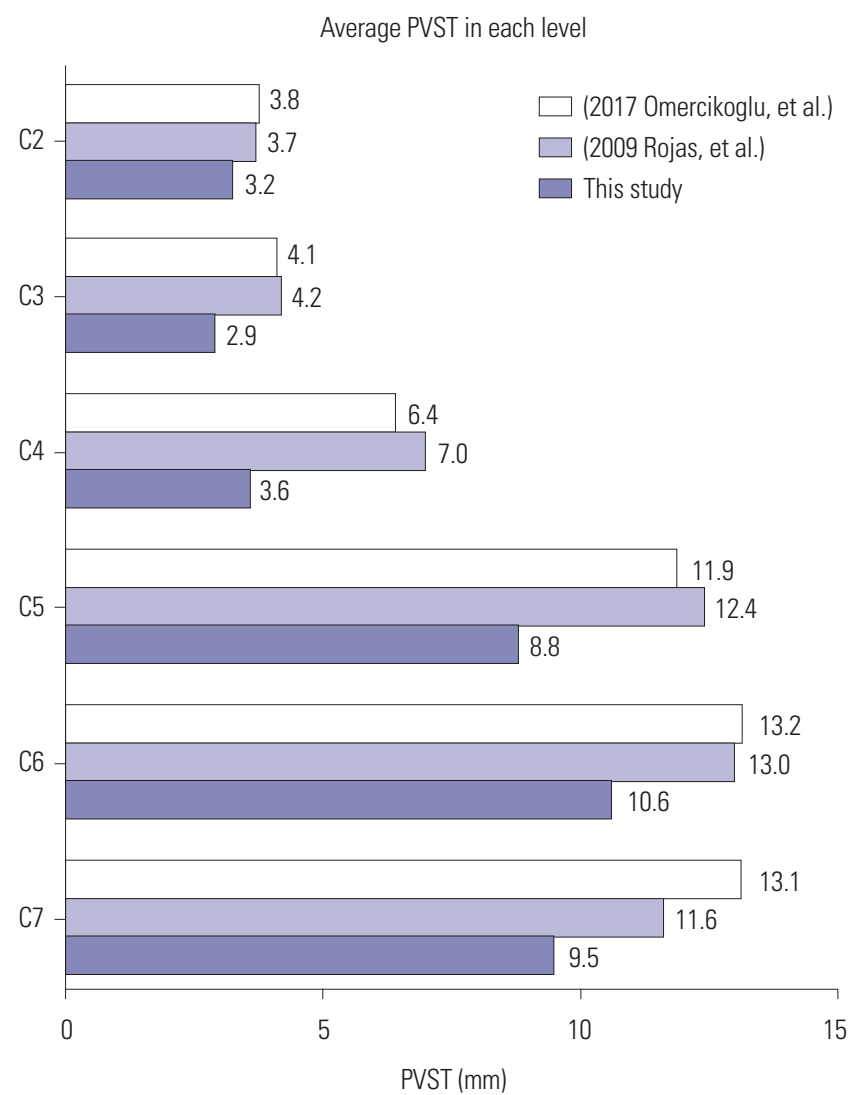

Fig. 4. Comparison of PVST with previous studies. Two previous studies suggested mean PVST values at each level for a healthy population (Omercikoglu et al., ${ }^{15}$ Rojas et al. ${ }^{16}$ ). PVST in our data was relatively lower than that of normal values due to the presence of anterior osteophytes. PVST, prevertebral soft tissue thickness. 

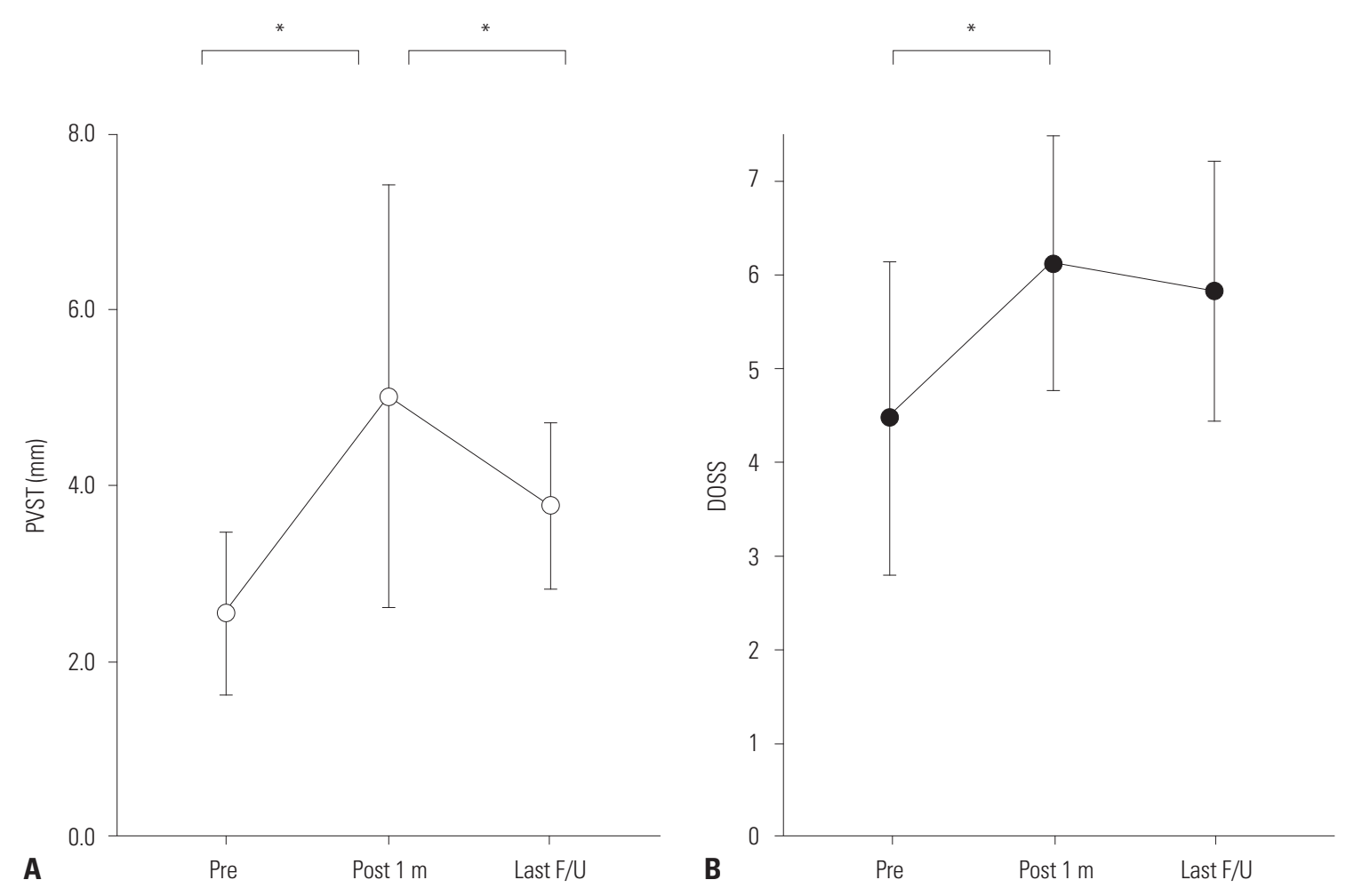

Fig. 5. Measurement of mean PVST and DOSS before and after the surgery $(n=17)$. (A) Mean PVST was $2.55 \pm 0.90$ mm preoperatively. However, it changed to $5.02 \pm 2.33 \mathrm{~mm}$ after 1 month and narrowed to $3.78 \pm 0.92 \mathrm{~mm}$ due to recurrence in some cases. These changes were all statistically significant $(p<0.001, p=0.016)$. (B) Likewise, DOSS scores were $4.47 \pm 1.61,6.12 \pm 1.32$, and $5.82 \pm 1.34$, respectively $(p=0.001, p=0.096)$. The graphs represent means and standard errors. * Statistical significance in paired t-test. DOSS, Dysphagia Outcome and Severity Scale; PVST, prevertebral soft tissue thickness.
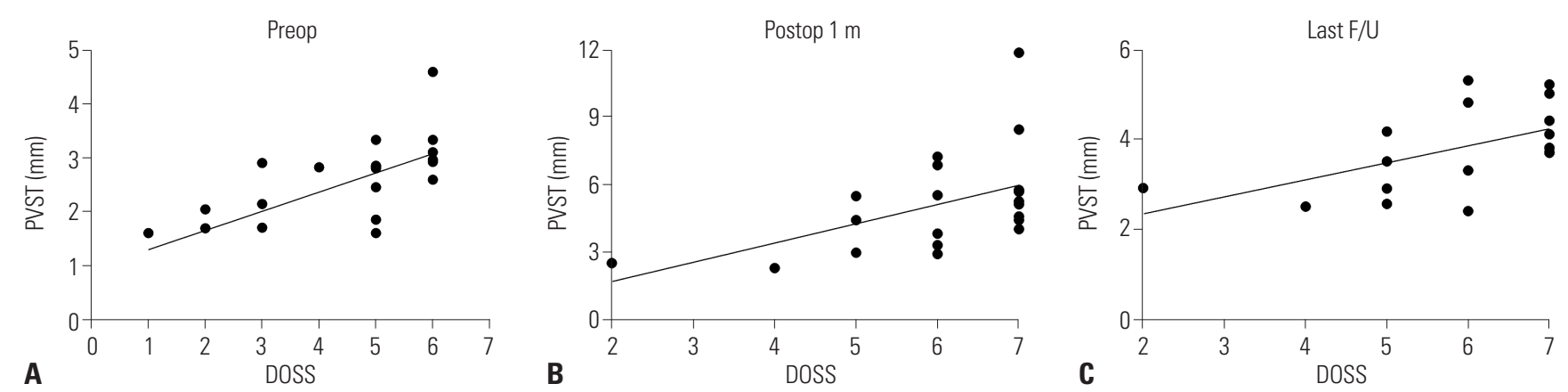

Fig. 6. Scatter plot of DOSS and PVST parameters. At each time point, a scatter plot is depicted, and Spearman's correlation test was performed. (A) Preoperatively $(n=21)$, the correlation coefficient $(\mathrm{R})$ value was $0.702(p<0.001)$. Postoperatively, $\mathrm{R}$ values were $0.539(p=0.012)$ after 1 month $(\mathrm{n}=21)(\mathrm{B})$ and $0.566(p=0.020)$ at last follow up ( $\mathrm{n}=17$ ) (C). DOSS, Dysphagia Outcome and Severity Scale; PVST, prevertebral soft tissue thickness.

\section{DISCUSSION}

Historically, Forestier and Rotes-Querol ${ }^{17}$ first described DISH as senile ankylosing hyperostosis in 1950. Resnick and Niwaya$\mathrm{ma}^{14}$ studied the radiographic and pathologic features of DISH for cadaveric and actual patients, and established diagnostic criteria that are commonly used now. Since the 1980s, there have been case reports in which severe cervical DISH triggered dysphagia due to direct mechanical compression of the esophagus. ${ }^{8,12,18-21}$ This condition was called DISH-related dysphagia, and Curtis, et al. ${ }^{8}$ first introduced the terminology as "DISHphagia." Most patients are men, with a predominance of 3:1 to
$6: 1,{ }^{11}$ and they are older than 60 years of age. In this study, all patients were men, and $81 \%$ were older than 60 years. Since 2009, several articles have reported on the results of anterior osteophytectomy in DISH-phagia, although the outcomes were inconsistent. Miyamoto, et al. ${ }^{10}$ reported a high recurrence rate in long-term follow-up (five of seven patients), whereas Urrutia and Bono, et al. ${ }^{12}$ and Jonathan, et al. ${ }^{22}$ achieved symptom relief without recurrence ( $\mathrm{n}=5$ and $\mathrm{n}=3$, respectively). However, no analytic paper has been published yet to predict surgical prognosis for DISH. Therefore, to our knowledge, this study is the first to use a large single-institutional database, and this is the first study seeking to predict the surgical outcomes of DISH- 
phagia through radiographic parameters.

In our study, four patients had AS or peripheral enthesopathy. The nature of new bone formation in DISH is thought to be similar in that of AS or peripheral enthesopathy. ${ }^{23}$ Nowadays, DISH and AS seem to not be mutually exclusive, rather they may involve common osteoblastic pathways, such as Wnt signaling and $\mathrm{NF} \kappa \mathrm{B}$ pathway, and can occur simultaneously. ${ }^{23,24}$ In that sense, clinicians should consider the possible presence of AS and peripheral enthesopathy in DISH-phagia patients and vice versa. Meanwhile, many patients $(61.9 \%, 13 / 21)$ experience ossification of the posterior longitudinal ligament (OPLL) or ossification of the ligamentum flavum (OLF) as comorbidities. Tsukahara, et al., ${ }^{25}$ a Japanese OPLL and DISH research group, proposed the idea that mutation of the collagen $6 \mathrm{Al}$ gene encoding the alpha chain of collagen type IV leads to osteoblast hyperactivity and hyperostosis of the vertebra. Further basic research is needed to investigate the common mechanisms of DISH, AS, OPLL, and OLF.

This study is the first study of the importance of soft tissue, not the osteophyte itself. Measuring osteophyte thickness is quite ambiguous because the cortical margin of the anterior vertebral body is not clear generally. Additionally, the osteophyte thickness is not proportionate to the severity of esophageal compression. Instead, measuring the narrowest PVST (PVL-T minus PVL-O) is not only simple and repeatable, but it also reflects the compression more intuitively. We identified a meaningful correlation between PVST and DOSS in all times, confirming that PVST is a good indicator for DISH-phagia. Therefore, we hypothesized that the narrowest preoperative PVST level is the most compressive level and that securing sufficient postoperative PVST is a key to surgery. The narrowest PVST location was above the C5 in all patients in this study; of these, 12 (57\%) were at C3. Moreover, PVST values in each level were narrower than those in healthy populations (Fig. 4). ${ }^{15,16}$ Until now, there have been many debates over which level of osteophyte is most contributable to dysphagia: C3/4 vs. C4 vs. $\mathrm{C} 4 / 5 .^{7,11,22}$ Because the transitional zone between the oropharynx and hypopharynx and the rigid anterior structure is located at C3 as hyoid bone and epiglottis, level C3 is thought to be the most problematic area. ${ }^{26}$ This was easily observed through VFSS or a laryngoscope.

We investigated how PVST changes postoperatively and its clinical relevance with dysphagia. Radiological and clinical improvement was achieved after 1 month, and the long-term clinical recurrence rate was quite low (3/17), thus suggesting surgical removal of DISH an efficient strategy for treating DISHphagia. Meanwhile, however, our results showed the possibility of recurrence regarding a decrease in PVST when it comes to long-term follow up (Fig. 5). While no additional surgery was performed in this study, reoperation might be considered if we do a study with larger population, due to the nature of ceaseless bony growth. To avoid additional operations and obtain a satisfactory result, surgeons should aim to attain as much prevertebral space as possible through the first surgery.

It is still debatable whether anterior support by the screw and plate system is necessary. According to a systematic review by Verlaan, et al., ${ }^{7}$ a simple osteophytectomy through an anterolateral approach is the most widely used surgical method. In contrast, von der Hoeh, et al. ${ }^{27}$ used a stand-alone polyetheretherketone cage in four patients and plate fixation in two patients. Although statistical analysis was not made due to the small numbers of plate fixation and fusion operation $(n=4)$, in our perspective, simple osteophytectomy alone showed more favorable results in DOSS change than plate fixation group ( $1.82 \pm 1.85$ vs. $1.00 \pm 0.00, p=0.511)$, and plate can sometimes trigger swallowing difficulty due to its volume effect. There are debates over whether anterior cervical plate fixation is useful. Some, including the present authors, consider that screw and plate fixation can cause additional esophageal compression, ${ }^{28}$ while others argue that a plate itself is not relevant to swallowing difficulties. ${ }^{29}$ Theoretically, a plate and screws would occupy space and contribute to PVST narrowing; however , it may also act as a physical barrier and suppress bony overgrowth. It is not certain that fixation surgery with a plate is superior or inferior to simple osteophytectomy in DISH-phagia. Further research with more patients and longer follow up is needed.

We found that a laryngoscope and VFSS provide good information for evaluating and diagnosing DISH-phagia. VFSS is effective for evaluating symptoms and level of swallowing failure. ${ }^{30}$ It is also useful as a follow-up study. Due to the lack of recognition of the usefulness of VFSS in the early period of this study, not all patients underwent VFSS preoperatively. In those cases, laryngoscope-guided diagnosis was made via consultation with otorhinolaryngologists. The laryngoscope shows great visualization of the actual compression and which level is the most problematic in patients with DISH-phagia, although dynamic visualization of swallowing is not feasible. Recently, a novel study suggested that combination of VFSS and esophagoscope is helpful to minimize the incomplete diagnosis in swallowing disorder. ${ }^{31}$ During surgery, the intraoperative C-arm can provide helpful information to determine the degree of bone drilling, both cephalocaudally and anteroposteriorly. In our cases, the use of C-arm fluoroscopy was not standardized in the initial period, and results were inconsistent, with relapse of dysphagia occurring, contrary to the favorable results when intraoperative C-arm monitoring was routinely used in the later period. We recommend careful surgery under the use of intraoperative fluoroscopic devices to confirm complete DISH removal, with a nasogastric tube inserted for checking the esophagus position.

This study has limitations, such as its retrospective design, and we failed to find various conditions and measurements other than PVST as a contributing factor for DISH-phagia. Because many different surgeons were involved independently, the lack of standardization of decisions on surgical procedure is another weak point. DISH-phagia is a rare disease and also 
difficult to diagnose, and applying a standardized procedure for a single surgeon or single center was nearly impossible. For the same reason, the regular examination of patients was not carried out, such that comparative analysis could not be clearly performed in the same period after the surgery. To overcome these limitations, standardized research is in progress, including regular follow-up every 6 months. In this study, we focused on gathering as many cases as possible to discover novel parameters reflective of surgical outcomes of DISH-phagia. Below the cricoid cartilage level (below C5), overestimation of the PVST occurs because the esophagus does not adhere to the trachea. In our study, this was ignorable because all patients had the "most problematic" level above the C5 upper endplate. In addition, unlike other studies that adopted the simple $0-3$ dysphagia scale, ${ }^{7,10,21,22}$ we used a seven-point scale (DOSS), which is more complex and has higher statistical power. Although some limitations exist, the authors are sure that this study will be helpful to understanding the nature of and predicting surgical outcomes for DISH-phagia, and we propose the clinical importance of PVST in patients with DISHphagia, rather than osteophyte thickness.

\section{AUTHOR CONTRIBUTIONS}

Conceptualization: Ho Yeol Zhang and Jeong-Yoon Park. Data curation: Young Soo Chung. Formal analysis: Young Soo Chung. Investigation: Young Soo Chung. Methodology: Jeong-Yoon Park. Project administration: Yoon Ha, Ho Yeol Zhang, and Jeong-Yoon Park. Resources: Yoon Ha, Ho Yeol Zhang, and Jeong-Yoon Park. Software: Young Soo Chung. Supervision: Yoon Ha, Ho Yeol Zhang, and Jeong-Yoon Park. Validation: Yoon Ha. Visualization: Young Soo Chung. Writing-original draft: Young Soo Chung. Writing-review \& editing: Jeong-Yoon Park. Approval of final manuscript: all authors.

\section{ORCID iDs}

Young Soo Chung https://orcid.org/0000-0001-8012-2902

Ho Yeol Zhang https://orcid.org/0000-0001-5005-1496

Yoon Ha https://orcid.org/0000-0002-3775-2324

Jeong-Yoon Park https://orcid.org/0000-0002-3728-7784

\section{REFERENCES}

1. Egerter AC, Kim ES, Lee DJ, Liu JJ, Cadena G, Panchal RR, et al. Dysphagia secondary to anterior osteophytes of the cervical spine. Global Spine J 2015;5:e78-83.

2. Mazières B. Diffuse idiopathic skeletal hyperostosis (ForestierRotes-Querol disease): what's new? Joint Bone Spine 2013;80:46670.

3. Yunoki M, Suzuki K, Uneda A, Okubo S, Hirashita K, Yoshino K. The importance of recognizing diffuse idiopathic skeletal hyperostosis for neurosurgeons: a review. Neurol Med Chir (Tokyo) 2016; 56:510-5.

4. Taljanovic MS, Hunter TB, Wisneski RJ, Seeger JF, Friend CJ, Schwartz SA, et al. Imaging characteristics of diffuse idiopathic skeletal hyperostosis with an emphasis on acute spinal fractures: self-assessment module. AJR Am J Roentgenol 2009;193(3 Suppl):
S20-4.

5. Aydin E, Akdogan V, Akkuzu B, Kirbaș I, Ozgirgin ON. Six cases of Forestier syndrome, a rare cause of dysphagia. Acta Otolaryngol 2006;126:775-8.

6. Kmucha ST, Cravens RB Jr. DISH syndrome and its role in dysphagia. Otolaryngol Head Neck Surg 1994;110:431-6.

7. Verlaan JJ, Boswijk PF, de Ru JA, Dhert WJ, Oner FC. Diffuse idiopathic skeletal hyperostosis of the cervical spine: an underestimated cause of dysphagia and airway obstruction. Spine J 2011;11: 1058-67.

8. Curtis JR, Lander PH, Moreland LW. Swallowing difficulties from "DISH-phagia". J Rheumatol 2004;31:2526-7.

9. Carlson ML, Archibald DJ, Graner DE, Kasperbauer JL. Surgical management of dysphagia and airway obstruction in patients with prominent ventral cervical osteophytes. Dysphagia 2011;26:34-40.

10. Miyamoto K, Sugiyama S, Hosoe H, linuma N, Suzuki Y, Shimizu K. Postsurgical recurrence of osteophytes causing dysphagia in patients with diffuse idiopathic skeletal hyperostosis. Eur Spine J 2009;18:1652-8.

11. Sebaaly A, Boubez G, Sunna T, Wang Z, Alam E, Christopoulos A, et al. Diffuse idiopathic hyperostosis manifesting as dysphagia and bilateral cord paralysis: a case report and literature review. World Neurosurg 2018;111:79-85.

12. Urrutia J, Bono CM. Long-term results of surgical treatment of dysphagia secondary to cervical diffuse idiopathic skeletal hyperostosis. Spine J 2009;9:e13-7.

13. O'Neil KH, Purdy M, Falk J, Gallo L. The Dysphagia Outcome and Severity Scale. Dysphagia 1999;14:139-45.

14. Resnick D, Niwayama G. Radiographic and pathologic features of spinal involvement in diffuse idiopathic skeletal hyperostosis (DISH). Radiology 1976;119:559-68.

15. Omercikoglu S, Altunbas E, Akoglu H, Onur O, Denizbasi A. Normal values of cervical vertebral measurements according to age and sex in CT. Am J Emerg Med 2017;35:383-90.

16. Rojas CA, Vermess D, Bertozzi JC, Whitlow J, Guidi C, Martinez CR. Normal thickness and appearance of the prevertebral soft tissues on multidetector CT. AJNR Am J Neuroradiol 2009;30:136-41.

17. Forestier J, Rotes-Querol J. Senile ankylosing hyperostosis of the spine. Ann Rheum Dis 1950;9:321-30.

18. Sobol SM, Rigual NR. Anterolateral extrapharyngeal approach for cervical osteophyte-induced dysphagia. Literature review. Ann Otol Rhinol Laryngol 1984;93(5 Pt 1):498-504.

19. Yee C, Wong HY, Fewer HD, Rogers AG. Two cases of dysphagia due to cervical spine osteophytes successfully treated surgically. Can Med Assoc J 1985;132:810-2.

20. Laus M, Malaguti MC, Alfonso C, Ferrari D, Zappoli FA, Giunti A. Dysphagia due to cervical osteophytosis. Chir Organi Mov 1995; 80:263-71.

21. Scholz C, Naseri Y, Hohenhaus M, Hubbe U, Klingler JH. Longterm results after surgical treatment of diffuse idiopathic skeletal hyperostosis (DISH) causing dysphagia. J Clin Neurosci 2019;67: 151-5.

22. Jonathan YC, Sayal P, Prezerakos G, Russo V, Choi D, Casey ATH. The surgical management of dysphagia secondary to diffuse idiopathic skeletal hyperostosis. Clin Neurol Neurosurg 2018;167: 36-42.

23. Mader R, Verlaan JJ, Buskila D. Diffuse idiopathic skeletal hyperostosis: clinical features and pathogenic mechanisms. Nat Rev Rheumatol 2013;9:741-50.

24. Kuperus JS, Waalwijk JF, Regan EA, van der Horst-Bruinsma IE, Oner FC, de Jong PA, et al. Simultaneous occurrence of ankylosing spondylitis and diffuse idiopathic skeletal hyperostosis: a systematic review. Rheumatology (Oxford) 2018;57:2120-8.

25. Tsukahara S, Miyazawa N, Akagawa H, Forejtova S, Pavelka K, 
Tanaka T, et al. COL6A1, the candidate gene for ossification of the posterior longitudinal ligament, is associated with diffuse idiopathic skeletal hyperostosis in Japanese. Spine (Phila Pa 1976) 2005;30: 2321-4.

26. Steele CM, Bailey GL, Chau T, Molfenter SM, Oshalla M, Waito $\mathrm{AA}$, et al. The relationship between hyoid and laryngeal displacement and swallowing impairment. Clin Otolaryngol 2011;36:30-6.

27. von der Hoeh NH, Voelker A, Jarvers JS, Gulow J, Heyde CE. Results after the surgical treatment of anterior cervical hyperostosis causing dysphagia. Eur Spine J 2015;24 Suppl 4:S489-93.

28. Shriver MF, Lewis DJ, Kshettry VR, Rosenbaum BP, Benzel EC, Mroz TE. Dysphagia rates after anterior cervical diskectomy and fusion: a systematic review and meta-analysis. Global Spine J 2017;
7:95-103.

29. Haws BE, Khechen B, Patel DV, Yoo JS, Guntin JA, Cardinal KL, et al. Swallowing function following anterior cervical discectomy and fusion with and without anterior plating: a SWAL-QOL (swallowing-quality of life) and radiographic assessment. Neurospine 2019;16:601-7.

30. Mattioli F, Ghirelli M, Trebbi M, Silvestri M, Presutti L, Fermi M. Improvement of swallowing function after surgical treatment of diffuse idiopathic skeletal hyperostosis: our experience. World Neurosurg 2020;134:e29-36.

31. Miles A, McMillan J, Ward K, Allen J. Esophageal visualization as an adjunct to the videofluoroscopic study of swallowing. Otolaryngol Head Neck Surg 2015;152:488-93. 